\title{
LOS BONOS PROYECTO EN LA FINANCIACIÓN DE INFRAESTRUCTURAS DE TRANSPORTE EN EUROPA
}

\author{
Autores: Eva Ballesté Morillas ${ }^{1}$ \\ Profesora Asociada \\ Departamento de Economía de la Empresa y Contabilidad, UNED \\ Rodrigo Martín García ${ }^{2}$ \\ Profesor Contratado-Doctor \\ Departamento de Economía de la Empresa y Contabilidad, UNED \\ $\mathrm{M}^{a}$ Teresa Corzo Santamaría ${ }^{3}$ \\ Profesora Titular \\ Departamento de Gestión Financiera, ICADE
}

\section{Resumen}

La dificultad para financiar las infraestructuras en Europa tras la crisis financiera global propició la búsqueda de mecanismos alternativos a los bancarios tra-

\footnotetext{
${ }^{1}$ eballeste@cee.uned.es

${ }^{2}$ rmarting@cee.uned.es

${ }^{3}$ mcorzo@icade.comillas.edu
} 
dicionales. En este trabajo se analizan los avances desarrollados para impulsar las infraestructuras prioritarias en el marco de la UE, a través de la colaboración entre administraciones, entidades multilaterales y agentes privados, principalmente con financiación privada de los proyectos, centrándose en los bonos proyecto como herramienta preponderante. Se analizan sus principales características, riesgos asociados, modalidades y se evalúan de forma crítica los avances realizados en la fase piloto, de cara al cumplimiento de sus objetivos (horizonte 2020).

Palabras clave: bonos proyecto; financiación de infraestructuras; Unión Europea; facilidades crediticias; elegibilidad de los proyectos.

\title{
Project bonds, promoters of investment in transport infrastructure in Europe
}

\begin{abstract}
The global financial crisis has caused difficulties in infrastructure financing in Europe, but also less-leveraged structures and ever-decreasing deadlines, which have stimulated the search for alternative mechanisms. In this paper, the main advancements to boost priority infrastructure within the European Union, through collaboration between governments, multilateral institutions and private agencies are analysed. Mechanisms that encourage greater private participation in project financing are described. The focus will be on project bonds, as a major tool: features, associated risks and modalities. We have studied the Pilot phase achievements, facing its horizon (2020).
\end{abstract}

Key words: project bonds; infrastructure financing; European Union; credit enhancements; project eligibility.

Recibido: 25/11/2015

Aceptado: 20/07/2016

\section{INTRODUCCIÓN}

El mundo de las infraestructuras en la actualidad se encuentra en un entorno complejo. Por un lado, las crecientes necesidades de nuevas infraestructuras ${ }^{4}$, coordinadas a nivel supranacional, continental e incluso intercontinental; y, por

\footnotetext{
${ }^{4}$ La OCDE (2007) fija las necesidades de inversión en infraestructuras (electricidad, transporte por carretera y ferroviario, telecomunicaciones y agua) en torno al 3,5\% del PIB mundial.
} 
otro, la dificultad para ser financiadas con estructuras financieras cada vez menos apalancadas y en plazos decrecientes, especialmente tras la crisis financiera global. Simultáneamente, los esfuerzos para la creación de nuevos proyectos deben combinarse con el mantenimiento de los existentes y su adaptación transnacional y a las nuevas necesidades de uso. Todo ello en un contexto de incapacidad presupuestaria de los agentes públicos para liderar los proyectos de obra civil, tan importantes en términos de producción y productividad ${ }^{5}$, y de agotamiento tanto del modelo convencional de financiación bancaria como de los instrumentos tradicionales de colaboración público-privada.

Ya en 2007, la OCDE, en su informe Infrastructure to 2030, ponía de manifiesto que "cerrar el gap de inversión en infraestructuras demandará enfoques innovadores, tanto en la búsqueda de financiación adicional como para el uso de las infraestructuras de manera más eficiente y más inteligente a través de las nuevas tecnologías, las estrategias de gestión de la demanda, los cambios regulatorios y la mejora de la planificación" (OCDE, 2007: 20). Resulta fundamental impulsar la inversión en infraestructuras, para lo que hay que avanzar en la búsqueda de nuevos mecanismos para la colaboración entre entidades públicas, privadas y organismos multilaterales, como el Banco Europeo de Inversión $(\mathrm{BEI})^{6}$ y el Banco Mundial.

La Unión Europea es muy consciente de la importancia de los transportes como medio de interconexión -como pone de manifiesto su programa Connect to compete $^{7}$ - para la generación de crecimiento, la creación de empleo, y la mejora de la calidad de vida. Cabe destacar que, en Europa, además, es un sector que impulsa de manera decidida la innovación. Durante el periodo presupuestario 2007-2013, se desarrolló un fuerte impulso en materia de infraestructuras. En el periodo presupuestario siguiente, 2014-2020, las infraestructuras de transporte serán uno de los ejes principales de la política de inversión europea. Sin embargo, para ello, se deberá contar con la apuesta pública decidida que establezca unos instrumentos claros e interesantes para el sector privado, deberá respaldar su estrategia con apoyo

\footnotetext{
${ }^{5}$ El Banco Mundial, en este sentido, publicó el informe "Valuing Infrastructure Stocks and Gains from Improved Performance" (1994), en el que establecía que, en promedio, un incremento de un 1\% en el stock de infraestructuras de una región, produciría un incremento de un $1 \%$ en el PIB.

${ }^{6}$ El Banco Europeo de Inversiones (BEI), creado por el Tratado de Roma en 1958, financia a través de préstamos a largo plazo, proyectos que contribuyen a la realización de los objetivos de la UE, y entre éstos, se consideran prioritarios las infraestructuras. El BEI se constituye como un importante emisor internacional de deuda soberana y entre sus principales características financieras, destacan las siguientes:

- El BEI financia su cartera de préstamos, principalmente, lanzando emisiones de obligaciones en los mercados internacionales de capitales.

- Los prestatarios del BEI cosechan las ventajas de la solidez financiera del banco cuya calificación crediticia es AAA.

- No persigue un fin lucrativo y presta en condiciones próximas al coste de su financiación.

${ }^{7}$ http://ec.europa.eu/transport/facts-fundings/connect-to-compete/index_en.htm.
} 


\section{Eva Ballesté Morillas, Rodrigo Martín García y Ma Teresa Corzo Santamaría}

económico y deberá contar con la coordinación entre administraciones europeas, nacionales y locales. Y es que solo la colaboración con el sector privado puede llevar a alcanzar los objetivos propuestos ya que permite proporcionar servicios de transporte de manera más eficiente que el modelo de financiación pública tradicional (Grout, 2003) y se beneficia de la mayor innovación y capacidad de gestión de los recursos de que están dotadas las empresas privadas frente al sector público (Spackman, 2002: 284), tan importante para los objetivos de la Unión Europea en $2013^{8}$.

El momento presente es óptimo, porque permite contar con un suficiente plazo temporal para conocer los avances alcanzados por esta iniciativa en términos de los dos objetivos propuestos inicialmente: promoción de proyectos relevantes de ámbito transnacional y la activación de mercados financieros de capitales para la financiación efectiva de los mismos. Las diferencias entre los datos cuantitativos y la valoración encontrada por parte de la UE y el BEI nos permitirá buscar un doble objetivo, donde se sitúa la principal aportación de nuestra contribución: 1) hacer una valoración crítica de estos logros; 2) realizar una propuesta de cara al horizonte de la iniciativa (2020) para optimizar esfuerzos organizativos y financieros de cara a su doble objetivo.

Así, el presente trabajo pretende contextualizar los bonos proyecto ${ }^{9}$ a nivel europeo para impulsar las infraestructuras en Europa, así como las iniciativas desarrolladas y los mecanismos que determinarán su éxito en los próximos años. Para ello, el resto de este documento se estructura como sigue: el apartado 2 recoge las medidas generales y el marco presupuestario en el que se ha desarrollado en el pasado la política de impulso de las infraestructuras y la situación programada para el próximo periodo presupuestario plurianual. El apartado 3 describe las medidas utilizadas hasta la actualidad para impulsar instrumentos financieros capaces de atraer inversores a los proyectos y que han supuesto la plataforma de lanzamiento de los bonos proyecto como mecanismo principal. El apartado 4 describe las características de los bonos proyecto, los riesgos asociados a estas operaciones, las modalidades previstas para su funcionamiento y las decisiones tomadas en relación con la elegibilidad de los proyectos de las redes transeuropeas de transporte para la aplicación de bonos proyecto. El apartado 5 muestra el resultado de la iniciativa hasta la actualidad en relación con los proyectos financiados, la captación efectiva de recursos financieros privados frente al capital público comprometido y el cumplimiento de los objetivos propuestos para la fase piloto de la iniciativa de bonos proyecto en Europa. Por último, el apartado 6, de conclusiones, constituye un análisis de la aplicación de

\footnotetext{
${ }^{8}$ En diciembre de 2013, la UE triplicó el presupuesto para innovación en transporte ferroviario, al considerarla el instrumento clave que permitirá "reducir los costes de servicio, aumentar la capacidad y ofrecer unos servicios ferroviarios más fiables y frecuentes para los clientes (http://ec.europa.eu/transport/ modes/rail/news/shift-to-rail_en.htm).

${ }^{9}$ No deben confundirse con los bonos de infraestructuras de la UE (cuyo propósito es elevar la financiación para un proyecto especial de la UE) ni con los eurobonos (bonos garantizados para financiar deuda pública).
} 
este instrumento al contexto de las infraestructuras europeas, su comparación con el uso dado en otras áreas y las variables que serán claves para su éxito para levantar capital suficiente para que el stock de infraestructuras europeo siga siendo un factor clave que impulse la competitividad y la equidad entre los integrantes de la UE.

\section{MEDIDAS GENERALES Y MARCO PRESUPUESTARIO}

La financiación privada de infraestructuras en Europa ha sido de naturaleza principalmente bancaria, al igual que en Oriente Medio y África. Esta modalidad de financiación gozaba, antes de la crisis financiera de 2008, de un excelente ambiente en términos de disponibilidad y precio (Dupas y otros, 2011:3). Sin embargo, desde entonces, la viabilidad de muchos proyectos con estructura tipo project finance se vio comprometida durante años. Así, en el periodo 2005-2012 -con una media anual de 76 proyectos analizados-, la financiación evolución europea de infraestructuras de nueva creación (greenfield) ${ }^{10}$ se estructuró mayoritariamente con fondos de la banca, resultando escasa la participación de los bonos y de capital aportado por fondos de inversión colectiva (Freshfields Bruckhaus Deringer, 2012: 6). Sin embargo, con independencia de la liquidez existente en el mercado, las restricciones de Basilea III para mejorar la capacidad del sector bancario para reducir los efectos de crisis financieras y su contagio entre economías, llevaron a reducir los plazos y las cantidades comprometidas en estos proyectos. El cambio de tendencia de financiación de infraestructuras de muy largo plazo y a un coste competitivo hacia estructuras soft miniperm ${ }^{11}$ y con bajo grado de apalancamiento conduce al sector de las infraestructuras a la búsqueda de nuevas alternativas para poder financiar los proyectos.

El paquete de medidas más importante en el ámbito de infraestructuras se denominó "Interconexión para Europa", se publicó el 19 de octubre de 2011 y materializó las propuestas del Marco Financiero Plurianual de 29 de junio de 2011 (Comisión Europea, 2011). Integró las siguientes iniciativas:

- Una Comunicación general sobre un Paquete de Crecimiento para unas Infraestructuras Europeas Integradas, un plan por valor de 50.000 millones de euros para impulsar las redes europeas energéticas, digitales y de transporte -conforme a la Estrategia Europa 2020 - y una nueva versión de las orientaciones en los tres ámbitos (transportes, energía y TIC).

\footnotetext{
${ }^{10} \mathrm{Greenfield} \mathrm{se} \mathrm{contrapone} \mathrm{a} \mathrm{los} \mathrm{proyectos} \mathrm{brownfield} \mathrm{o} \mathrm{preexistentes.}$

${ }^{11}$ Estructuras con una duración entre 3 y 5 años -más cortas, por tanto, que la financiación a largo plazo-que se emplean para proyectos de colaboración público privada. Se utilizan habitualmente como instrumentos puente para el acceso a financiaciones de carácter más permanente (a vencimiento de proyecto).
} 
- Una Comunicación sobre la fase piloto de la Iniciativa Europa 2020 de bonos para la financiación de proyectos (gestionada por el Banco Europeo de Inversiones), que se previó para 2012.

- Una Comunicación sobre un marco para la próxima generación de instrumentos financieros innovadores: plataformas de la UE de capital y deuda.

- Una propuesta de Reglamento por el que se creó el Instrumento de Interconexión para Europa.

- Una propuesta de Reglamento que estableció las directrices para articular la Red Transeuropea de Transporte (RTE-T ${ }^{12}$ ).

- Una propuesta de Reglamento sobre directrices para la infraestructura transeuropea de energía (TEN-E).

- Una propuesta de Reglamento por el que se establecen directrices sobre redes de telecomunicaciones transeuropeas.

Este conjunto de medidas puso de manifiesto la importancia de contar con un marco reglamentario mejorado de instrumentos financieros ${ }^{13}$ capaces de atraer inversores a los proyectos de colaboración público privada y supuso un primer paso para solucionar la financiación de grandes proyectos de infraestructuras. El reparto de recursos mostró claramente tanto líneas estratégicas como el peso relativo de cada una de ellas. Así, los sistemas de transporte constituyeron la partida más importante en el reparto, con 31.700 millones de euros (63,4\% del presupuesto); energía asumió un presupuesto de 9.100 millones de euros (18,2\%); y el apoyo a las redes digitales de alta velocidad recibió 9.200 millones de euros (18,4\%). Así se definió y se asignaron las dotaciones económicas para lo que se consideró la "columna vertebral de Europa" (Gerebizza y Tricarico, 2013). El presupuesto para el periodo 2014-2020, aprobado en diciembre de 2013, ratificó estas cantidades propuestas inicialmente para la interconexión de Europa. En el caso de la RTE-T, las prioridades generales fueron (Champion, 2016: 2): 1) generar tramos transfronterizos; 2) la reducción de la diferencia de calidad de infraestructuras de transporte entre los Estados miembros; 3) impulsar la intermodalidad; 4) la reducción de emisiones del transporte.

\section{MEDIDAS PARA IMPULSAR INSTRUMENTOS FINANCIEROS CAPACES DE ATRAER INVERSORES A LOS PROYECTOS}

\footnotetext{
${ }^{12}$ La política RTE-T busca una mejor coordinación de la planificación y el desarrollo de la red de transportes a nivel europeo. A tal fin, la Comisión Europea ha creado el mecanismo denominado "Conectar Europa" destinado a construir las infraestructuras que se consideran prioritarias para una mejor cohesión y vertebración de Europa. Es este sentido, cabe destacar la importancia de los corredores de la red principal europea como plataforma para la implementación de una red de transporte multimodal para Europa.

${ }^{13}$ En la línea de lo puesto de manifiesto en el Libro Blanco. Hoja de ruta hacia un espacio único europeo de transporte: por una política de transportes competitiva y sostenible, p. 17.
} 
En este complejo contexto, ha sido necesario buscar mecanismos alternativos que permitan canalizar recursos hacia proyectos de infraestructuras. La principal dificultad parte del rechazo de la banca a asumir el mismo perfil de riesgo aceptado hasta la crisis, perfil que las administraciones públicas (concedentes de los proyectos) no pueden soportar.

Así, la Unión Europea diseñó una batería de instrumentos encaminados a proporcionar las garantías que el ente concedente y la banca no estaban dispuestos a asumir. La solución propuesta consistió en la aportación de capital en tres niveles: recursos propios (capital -equity), capital riesgo, e instrumentos de deuda (préstamos, garantías a intermediarios o mecanismos de reparto de riesgos con instituciones financieras). En concreto, se propusieron tres instrumentos ${ }^{14}$ que se aplicarían a los proyectos de la Red Transeuropea de Transporte (RTE-T) y cuya finalidad se sintetiza en la Tabla 1:

\section{Tabla 1. Instrumentos aplicables a los proyectos de la Red Transeuropea de Transporte (RTE-T)}

\begin{tabular}{|l|l|l|}
\hline \multicolumn{1}{|c|}{ FINALIDAD } & \multicolumn{1}{c|}{ MECANISMO } & \multicolumn{1}{c|}{ INSTRUMENTO } \\
\hline $\begin{array}{l}\text { Un instrumento para participar } \\
\text { en el capital de los proyectos, } \\
\text { complementando el conjunto de } \\
\text { herramientas existentes "con el } \\
\text { objetivo de desarrollar aún más } \\
\text { los mercados de capital de riesgo } \\
\text { en toda la UE"15. }\end{array}$ & $\begin{array}{l}\text { Participar en los } \\
\text { recursos propios de las } \\
\text { sociedades responsables } \\
\text { de los proyectos }\end{array}$ & El fondo Marguerite \\
\hline $\begin{array}{l}\text { Instrumentos "para responder } \\
\text { a las necesidades de múltiples } \\
\text { modelos de financiación aplicados } \\
\text { en toda la UE, el tamaño y el } \\
\text { sector de los proyectos y la etapa } \\
\text { de desarrollo de la financiación } \\
\text { del proyecto y los mercados de } \\
\text { capitales" }\end{array}$ & $\begin{array}{l}\text { Coberturas para la } \\
\text { compartición de riesgos }\end{array}$ & $\begin{array}{l}\text { El instrumento de garantía } \\
\text { de crédito (Loan Guarantee } \\
\text { Instrument) para proyectos de la } \\
\text { red transeuropea de transporte }\end{array}$ \\
\cline { 2 - 3 } & Bonos & $\begin{array}{l}\text { Los bonos proyecto de la Unión } \\
\text { Europea }\end{array}$ \\
\hline
\end{tabular}

Fuente: elaboración propia.

El fondo Marguerite es un fondo constituido por inversores públicos y privados (incluida la Comisión Europea) para la financiación de proyectos a largo plazo de nueva construcción (tipo greenfield) e impulsando el cumplimiento de los objetivos de política pública, sin olvidar los principios de mercado. Participa en

${ }^{14}$ Estas herramientas, en materia de infraestructuras, son análogas a las propuestas para impulsar la competitividad, reforzar a las Pymes, promover el autoempleo, microempresas y empresas sociales y también en el área de educación y cultura, con adaptaciones en función del destinatario programado.

${ }^{15} \mathrm{Op}$. cit. 
el capital social de los proyectos nacionales o transnacionales, con una inversión mínima de 10 millones de euros y un máximo del 10\% del total del fondo (actualmente de 710 millones: 100 millones de cada patrocinador -Francia, España, Italia, Alemania, Polonia y el Banco Europeo de Inversiones-, 80 millones de la Comisión Europea y 30 millones de otros inversores $)^{16}$, para financiar proyectos de transportes y energía elegibles por la Comisión, en el marco de la UE28. A pesar del importante esfuerzo a lo largo del bienio 2014-15 que llevó a que la inversión total desde su constitución (2010) hasta finales de 2015 superase los 400 millones de euros, repartida en un total de 11 proyectos en 9 países, no ha sido el instrumento decisivo que pretendía participando en el capital de los proyectos, llegando a propiciar la captación de financiación en los mercados no superior a los 3.000 millones de euros (Marguerite, 2016: 8), repartidos en proyectos de nueva creación (cerca del 65\%) y proyectos antiguos (35\%).

El instrumento de garantía de crédito para proyectos de la red transeuropea de transporte (LGTT -Loan Guarantee Instrument for Trans-European Transport Network Projects) es un instrumento financiero desarrollado por la Comisión Europea y el BEI para estimular la participación privada en la financiación de infraestructuras de la Red Transeuropea de Transporte (RTE-T). Creado en 2008, está dotado de 1.000 millones de euros y tiene previsto facilitar hasta un máximo de 20.000 millones en deuda preferente de proyectos de transporte, financiando un máximo del 10\% de la cuantía total de la deuda preferente ${ }^{17}$. Mejora la capacidad del prestatario para atender al servicio de la deuda preferente durante el período inicial de la explotación del proyecto (fase de aceleración) mientras no se haya estabilizado el nivel de ingresos. Ha sido concebido para mejorar la calidad de crédito global del proyecto y justificar una reducción de los márgenes de riesgo aplicados a los préstamos prioritarios. Hasta finales de 2014 ha impulsado una inversión total de 12.000 millones en Francia, Alemania, Portugal, España y el Reino Unido (Comisión Europea, 2015b: 19). Sin embargo, aún no ha probado ser capaz de soportar por sí mismo el logro de la promoción de los proyectos considerados prioritarios (EY, 2015: 44).

\section{LOS BONOS PROYECTO DE LA UNIÓN EUROPEA}

El modelo tradicional de impulso y financiación de infraestructuras, ante un proyecto licitado por una administración pública, partía de una propuesta de ejecución-operación, por parte de una empresa o grupo de empresas, para lo que constituía una entidad especial como vehículo (SVP) para su desarrollo, constituyéndose como accionistas-sponsors de la misma. Los recursos propios de la

\footnotetext{
${ }^{16}$ PWC, 2014.

${ }^{17}$ Permite un máximo del 20\% en supuestos excepcionales.
} 
entidad creada al efecto constituían entre el 10\% y el 20\% del total de recursos necesarios para la ejecución del proyecto y el resto era financiado por medio de préstamos bancarios, como se refleja en la Figura 1. Este funcionamiento seguía una estructura del tipo project finance y el agotamiento de este modelo es lo que ha llevado a los poderes públicos a buscar alternativas de mercado más adecuadas a la situación actual y a las necesidades de creación de nuevas infraestructuras.

\section{Figura 1. Modelo de financiación de infraestructuras tipo project finance}

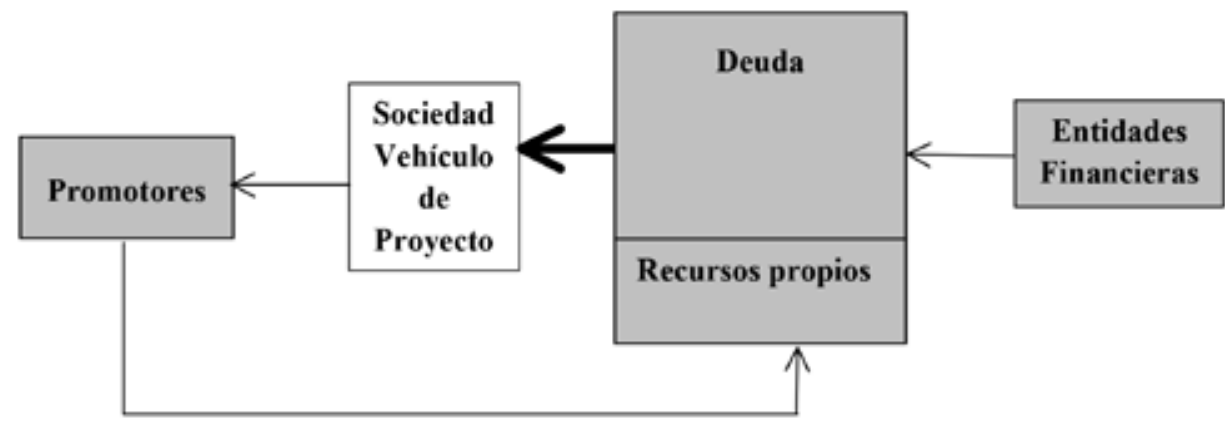

Fuente: elaboración propia.

La Iniciativa Europa 2020 Project Bond busca proporcionar una nueva alternativa a la participación privada en la generación de infraestructuras. La Comisión Europea y el BEI firmaron en noviembre de 2012 el acuerdo para poner en marcha la fase piloto (reglamento de la Unión Europea No 670/2012 del Parlamento Europeo y del Consejo, de 11 de julio de 2012), con los fines de atraer financiación privada a los proyectos y de permitir la emisión de la deuda en los mercados de capitales, animando a inversores institucionales a participar en este tipo de proyectos.

En el marco de una estrategia global de financiación privada de infraestructuras, los bonos proyecto han sido considerados por las instituciones, desde su lanzamiento, como uno de los instrumentos con mayor interés, por considerarse que contaban con mayor potencial de captación de recursos procedentes de la inversión privada y, por tanto, de dinamización de la actividad de impulso de generación de una red transnacional de infraestructuras de transporte y de activación de los mercados financieros en lo relacionado con proyectos de esta naturaleza.

Veamos a continuación cuál es la estructura financiera de los esquemas financiados con bonos proyecto de forma comparada con los instrumentos tradicionales; cuáles son los riesgos, tanto los vinculados a los proyectos que financian, como los intrínsecos a su naturaleza financiera; las modalidades que se han empleado en el marco de la iniciativa europea; y, por último el calendario de ejecu- 
ción-financiación de un proyecto tipo, de forma comparada entre las operaciones tradicionales y las distintas posibilidades de colocación de los bonos proyecto.

\subsection{Estructura financiera de los esquemas financiados con bonos proyecto}

En el modelo de financiación mediante bonos proyecto, las compañías que constituyen la sociedad vehículo de proyecto participan en su capital (entre el 10 $\%$ y $20 \%$ del total) y el resto es un esquema de deuda, conformado por:

- Una emisión de bonos proyecto, cuya calificación crediticia, para poder ser colocada en los mercados de capitales, debería ser triple A, negociable en mercados organizados y susceptible, por tanto, de adquisición por parte de inversores institucionales. Esta deuda cuenta con calificación senior, es decir, cuenta con derecho preferencial en la cascada de cobros de la sociedad vehículo de proyecto ante un problema de liquidez.

- Una participación del BEI en el proyecto mediante la concesión de un préstamo o una garantía a la sociedad, por un importe variable, determinado proyecto a proyecto, pero nunca superior al $20 \%$ del total de deuda senior, cubriendo los déficits de financiación durante el período de construcción y los riesgos relacionados con la generación de flujos de caja una vez que el proyecto se encuentra operativo.

Esta aportación del BEI, cuando tome forma de préstamo o línea de crédito, tendrá carácter subordinado con respecto a los bonos proyecto. Por tanto, independientemente de la fórmula empleada (préstamo o garantía), favorecerá la probabilidad de pago al resto de acreedores, mejorando así la calificación crediticia del emisor y facilitando la obtención de la calificación crediticia objetivo que posibilitaría el éxito de la colocación en el mercado de la deuda senior ${ }^{18}$.

El BEI se encontraría, en la lista de prelación de cobros, detrás de toda la deuda senior, pero por delante de los préstamos participativos, del resto de deuda subordinada y, por supuesto, de los pagos al capital ${ }^{19}$.

${ }^{18}$ El Banco Europeo de Inversiones, al igual que la banca comercial, exige una serie de garantías habituales para este tipo de proyectos y que cubren cada una de las obligaciones asumidas bajo los créditos senior, entre las que se encuentran:

- Prenda de todos los derechos, importes e indemnizaciones que recibiera por parte de cualquier asegurador conforme al plan de seguros del proyecto.

- Prenda de los derechos de crédito derivados del contrato de CPP.

- Prenda de las cuentas del proyecto.

- Prenda de los derechos de crédito derivados de los contratos de cobertura de tipo de interés.

- Prenda de los derechos derivados de los contratos de construcción.

${ }^{19}$ La participación del BEI como garante en estas operaciones establece una contrapartida (fee) a satisfacer por la SVP, de conformidad con las tablas fijadas por esta institución. 
Se comprueba, por tanto, que la proporción recursos propios/deuda es equivalente al modelo anterior, sustituyendo la financiación bancaria por recursos públicos (participación del BEI) y privados (bonos). La financiación pública se obtiene, dado el horizonte temporal de la iniciativa, en dos fases: la primera, en el marco financiero plurianual en el cual fue diseñada (2007-2013) y la segunda, en el marco financiero plurianual presente (2014-2020). En esta prueba piloto, los proyectos elegibles debían alcanzar el cierre financiero antes de 2016, lo que hace posible una evaluación temprana de los resultados de la iniciativa.

\subsection{Riesgos asociados a las infraestructuras de transporte y modalidades de bonos proyecto}

La naturaleza de los proyectos (nuevos vs. preexistentes) determina los factores de riesgo que afectarán a los mismos y las estructuras financieras necesarias para promoverlos y esto tendrá, como se verá más adelante, importantes implicaciones. Los factores de riesgo vinculados a proyectos de infraestructuras son los siguientes:

- El riesgo de construcción. El proyecto puede ser abandonado durante su construcción. Factores como la complejidad de los trabajos, la disponibilidad para reemplazar al contratista en caso de quiebra y contar con fondos contingentes suficientes para cubrir el incremento de coste provocado por los retrasos en la obra son riesgos durante la fase de construcción de la infraestructura a considerar. Lógicamente, este es un riesgo que afecta únicamente a los proyectos de nueva creación.

- El riesgo de ramp-up. El período de ramp up es el tiempo que transcurre desde la entrada en servicio de la infraestructura hasta que la utilización se consolida por completo y es posible estimar de forma fiable los flujos que generará en el futuro. Lógicamente, existe para los proyectos nuevos, con riesgo de demanda. En cambio, para los proyectos preexistentes sí existe una base ya testada y, si no se producen cambios significativos (creación de infraestructura alternativa, abandono o depresión de la zona o región a que proporciona servicio, etc.), este riesgo no sería significativo.

- El riesgo de garantía. Las entidades constituidas específicamente para un proyecto y que dependen del mismo para su viabilidad, pueden verse significativamente afectadas si los precios de financiación y garantías son excesivamente altos. Por ejemplo, si consideramos un proyecto de Colaboración Público-Privada basado en pagos por disponibilidad y cuya clasificación crediticia es de $\mathrm{BBB}$, es poco probable que la calificación de su deuda tenga una calificación crediticia superior a esa triple B (al menos que haya un fuerte respaldo ante posibles casos de incumplimiento). Este riesgo afecta tanto a proyectos nuevos como preexistentes, pero habitualmente es mayor para los primeros. 
- El riesgo operativo. Algunos proyectos tienen un fuerte componente de riesgo operativo, bien en términos de coste o bien en términos de capacidad de generación de flujos de caja. En estos casos, es difícil asegurar el reemplazo del operador debido a la falta de alternativas para mejorar ante un escenario desfavorable. Como ocurre con el anterior, afecta tanto a proyectos nuevos como preexistentes, pero suele ser más alto para los primeros.

- El riesgo soberano. La calificación crediticia para los proyectos de la Unión Europea teóricamente debe ser "AAA". No obstante en la práctica, no es posible desconectar el perfil de riesgo de crédito de un proyecto del riesgo del país al que pertenece. Afecta, lógicamente, a todos los proyectos.

El diseño de cada operación debe tomar en consideración estos riesgos, así como la participación pública por parte de las instituciones, pero también los mecanismos que pueden mitigar sus efectos, (como se puede ver en Rossi y otros, 2015: 106-108). Así, para la financiación de estos proyectos, el Banco Europeo de Inversiones diseñó dos modalidades de colaboración: financiada y no financiada (BEI, 2012: 10), en función de la naturaleza de su aportación:

1. PBCE financiada: el BEI proporciona financiación a la sociedad adjudicataria en forma de tramo de deuda subordinada (similar a las estructuras mezzanine) con el límite del 20\% del importe del tramo de bonos senior vinculados (ver Figura 2).

\section{Figura 2. Modalidad PBCE financiada}

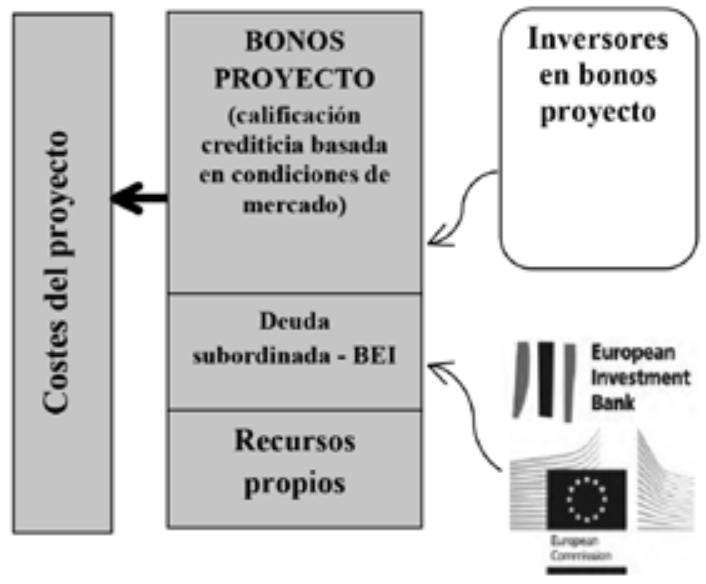

Fuente: elaboración propia, con base en los esquemas de funcionamiento del Banco Europeo de Inversiones (BEI, 2012:10). 
El impacto de esta modalidad financiada para los tenedores de bonos proyecto en dos ámbitos se produce en forma de:

- Mejora de la calidad del riesgo. Reduce el riesgo de impago al facilitar recursos en caso de insuficiente generación de efectivo por parte del proyecto.

- Reducción del riesgo de construcción y operación por su carácter subordinado.

- Mejora del ratio de cobertura al servicio de la deuda (RCSD).

2. PBCE no financiada: el BEI proporciona una Carta de Crédito revolvente a largo plazo asociada al proyecto, cuyo beneficio se asignará a quien figure como garante (trustee) ${ }^{20}$ (ver Figura 3).

Su carácter rotativo permite usar este mecanismo como una línea de crédito disponible para la SVP en el caso de que los flujos de caja generados por el proyecto no sean suficientes para la finalización de la construcción o para garantizar el RCSD comprometido para los bonos proyecto.

Figura 3. Modalidad PBCE NO financiada

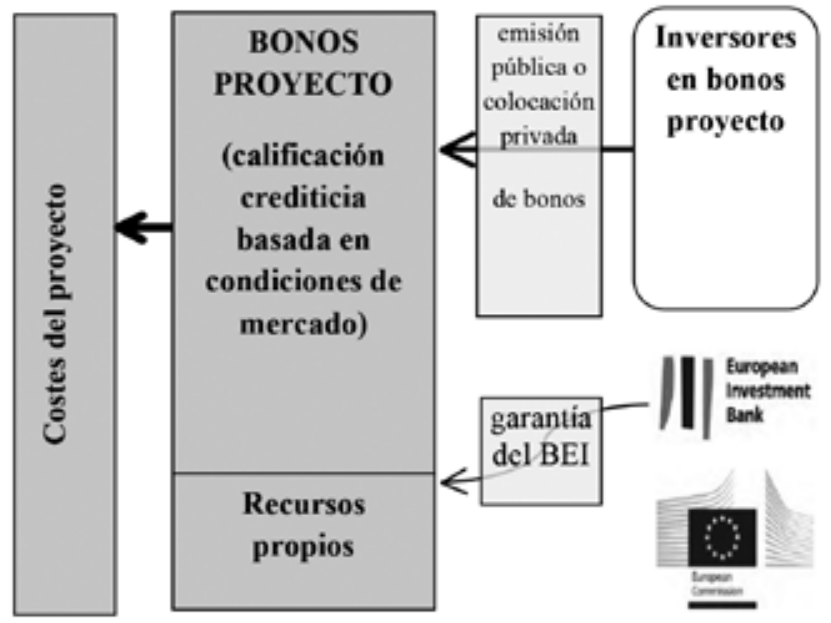

Fuente: elaboración propia, con base en los esquemas de funcionamiento del Banco Europeo de Inversiones (BEI, 2012: 12).

\footnotetext{
${ }^{20} \mathrm{El}$ encargado de representar y proteger los intereses del colectivo de tenedores de los Bonos Proyecto.
} 
La financiación mediante bonos proyecto solo es aplicable a aquellos proyectos que sean elegibles para el BEI y por tanto deben cumplir con los objetivos e intereses marcados por la UE. Las prioridades en la elección de proyectos se fijan en función de la contribución al crecimiento, empleo, cohesión regional y sostenibilidad medioambiental en Europa y la elección de los proyectos obedece principalmente a dos criterios: la relevancia y la realización del proyecto.

- La relevancia es el primer valor añadido del proyecto. Solamente aquellos proyectos que cumplen con las políticas marcadas por la UE pueden ser elegibles.

- Por realización se entiende la capacidad del proyecto en términos de efectividad, eficiencia, sostenibilidad e impacto medioambiental.

Las áreas declaradas prioritarias han sido:

- Los Proyectos Prioritarios de la RTE-T.

- Las autopistas del mar, como alternativa a las carreteras congestionadas.

- Sistemas de gestión del tráfico ferroviario europeo (ERTMS), para la interoperabilidad de la red ferroviaria europea.

- Gestión del tráfico aéreo, modernización y espacio aéreo europeo.

- Los sistemas Inteligentes de Transporte y los destinados a impulsar la interoperabilidad y la continuidad de la promoción del tráfico en tiempo real y los viajes los servicios de información europeos.

\subsection{El proceso de financiación mediante bonos proyecto frente al sistema tradicional}

Para materializar la colaboración público privada en los proyectos, cualquiera que sea el mecanismo de financiación, la operación comienza con la solicitud de la institución que se constituye como promotora del proyecto. Una vez que éste ha demostrado ser elegible -tanto por el cumplimiento de las pautas de la UE como los criterios del BEI- se fijan los criterios de financiación, que deben ser asesorados por este último, en base a cuestiones de viabilidad económico-financiera, técnicas y medioambientales. Este triple criterio debe cumplirse de cara a la emisión de deuda o garantía que constituye la participación pública (BEI) en el proyecto (Figura 4). 
Figura 4. Ciclo de la participación del BEI en un proyecto de financiación

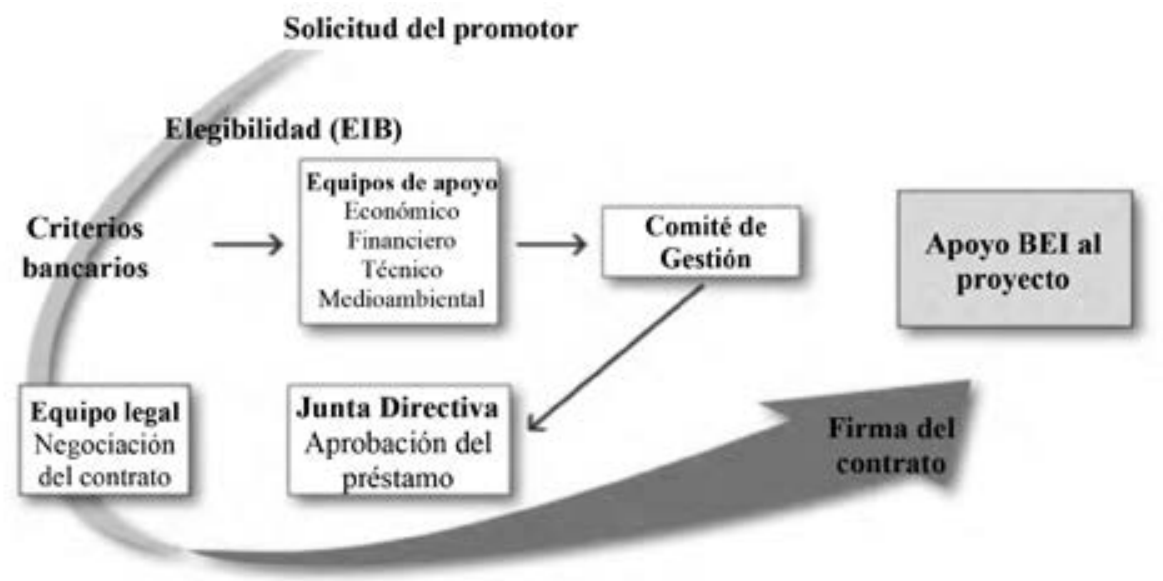

Fuente: Banco Europeo de Inversiones (2016). http://www.eib.org/projects/cycle/

En cualquier caso, todo este esquema debe ajustarse a los plazos implícitos en cualquier operación financiera de esta naturaleza. El calendario en una operación de financiación tradicional de esta naturaleza supera habitualmente los dos años, desde el momento del anuncio hasta la firma definitiva del contrato, que posibilita el comienzo de la ejecución del proyecto (Figura 5). 
Figura 5. Calendario de un proyecto de infraestructuras con financiación bancaria (préstamo)

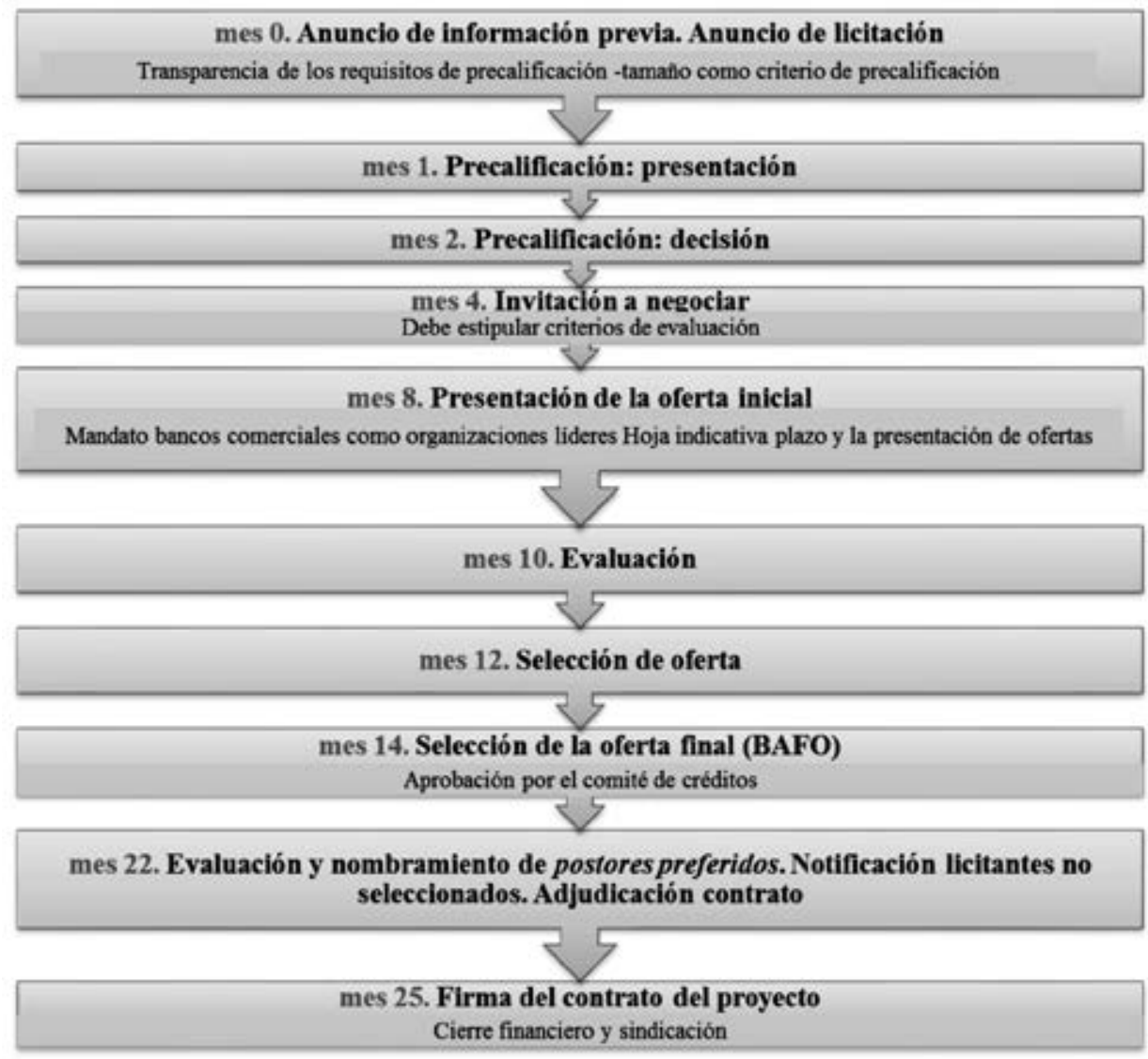

Fuente: EPEC y Freshfields Bruckhaus Deringer (2013: 6-15). Adaptación.

La duración del proceso para la financiación de los proyectos no puede supeditarse al instrumento financiero empleado para ello, sino que son los trámites administrativos quienes marcan el calendario, debiendo encajarse las iniciativas para la financiación de los proyectos en el marco administrativo. En el nuevo sistema, los bonos proyecto sustituyen dicha financiación bancaria de forma integrada en la tramitación administrativa que constituye el marco legal aplicable. En el caso de utilización de bonos proyecto, en las modalidades financiada o no financiada por parte del BEI, la colocación de bonos puede ser privada o pública y sus líneas temporales son las que se muestran en Figura 6 y Figura 7. 
Figura 6. Calendario de un proyecto de infraestructuras con emisión de bonos proyecto y colocación privada

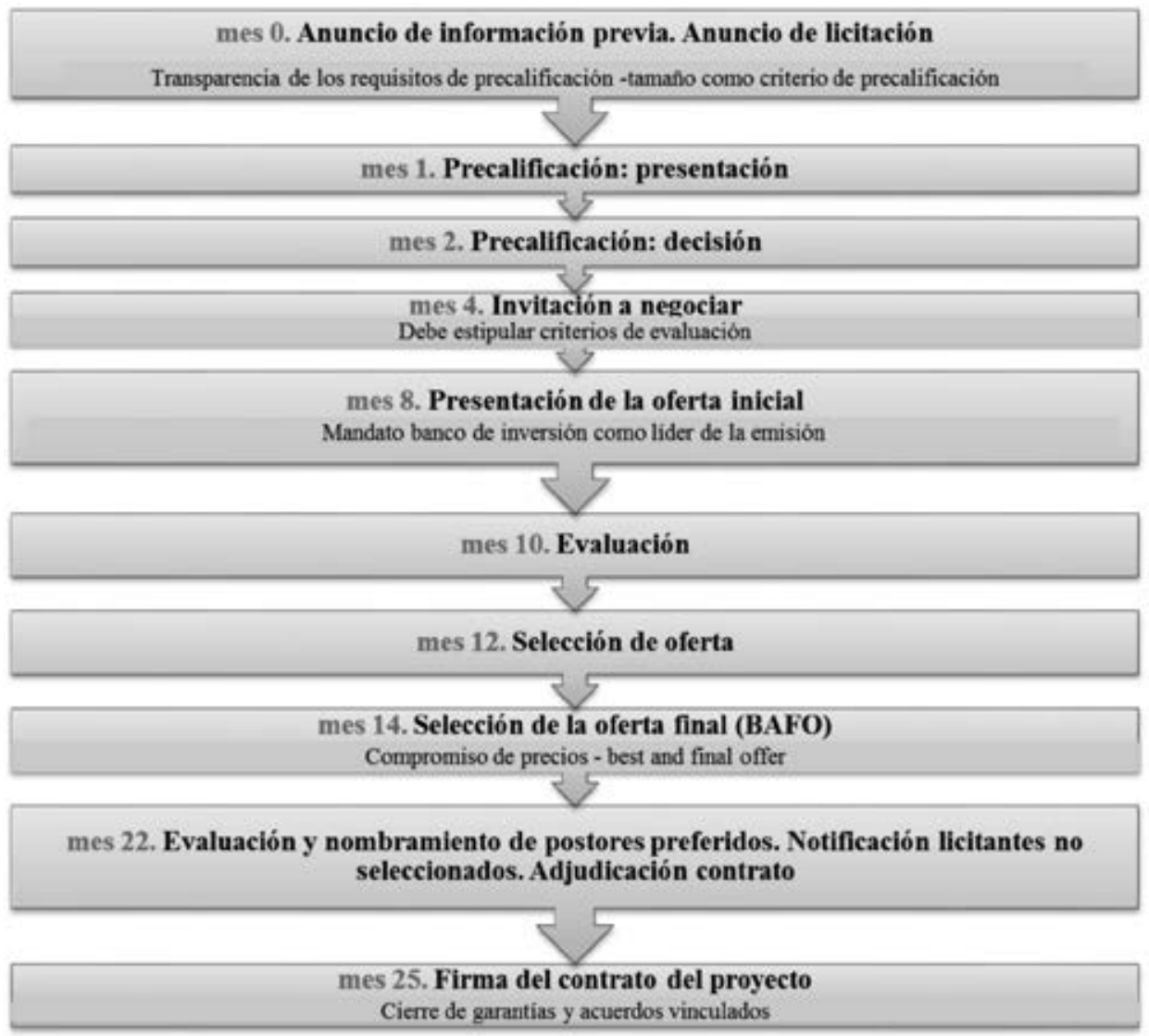

Fuente: EPEC y Freshfields Bruckhaus Deringer (2013: 6-15). Adaptación. 
Figura 7. Calendario de un proyecto de infraestructuras con emisión de bonos proyecto y colocación pública

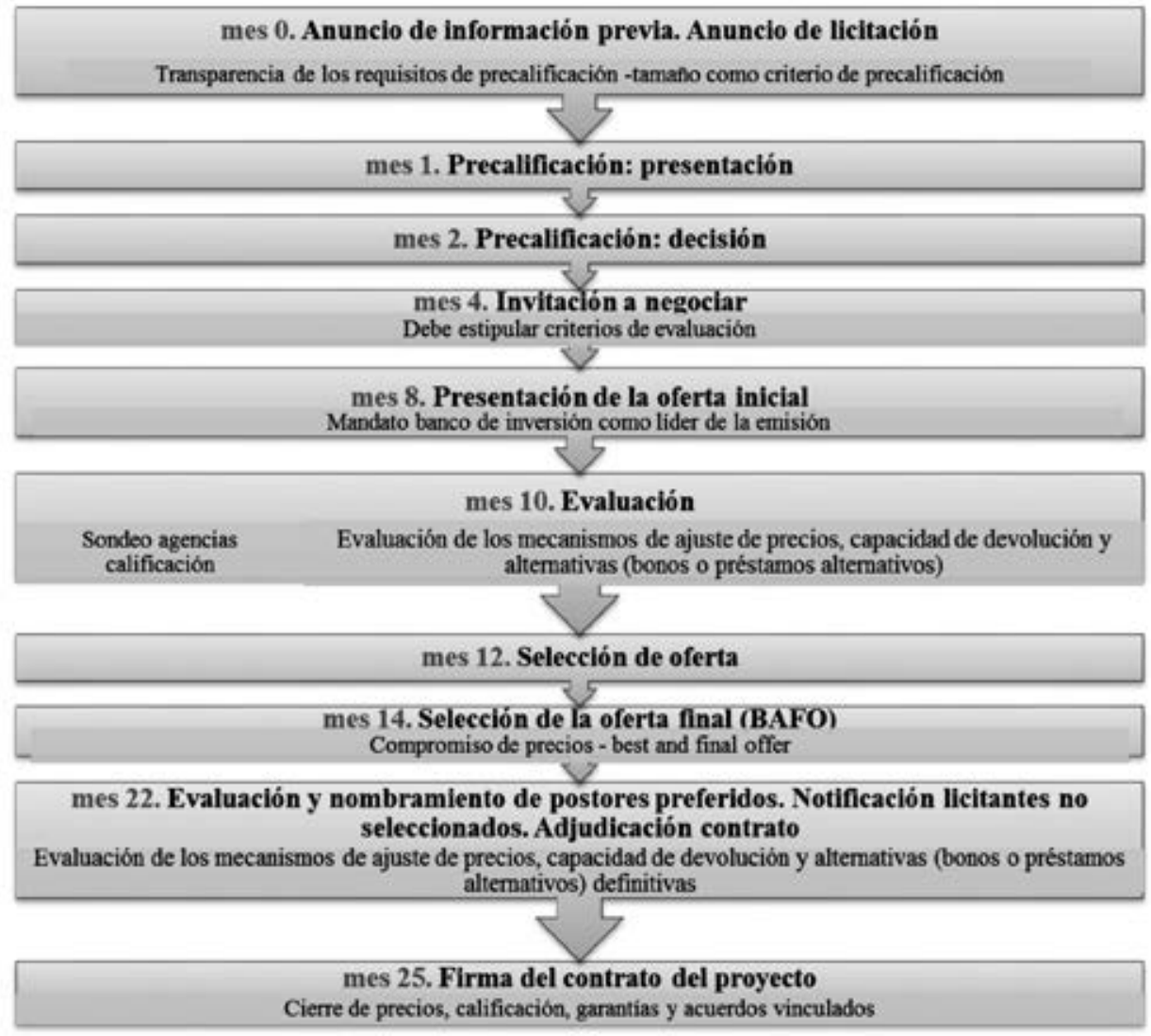

Fuente: EPEC y Freshfields Bruckhaus Deringer (2013: 6-15). Adaptación.

Como puede verse, los tres procedimientos tienen el mismo horizonte temporal y únicamente se encuentran diferencias menores en los meses 8, 10,14, 22 y 25 del proceso:

- En el momento de la presentación de la oferta inicial (mes 8), se produce el mandato de la entidad (consorcio) que se compromete a financiar el proyecto (banco comercial, banco de inversión, líder del sindicato bancario o líder de la emisión de bonos - públicos o privados-), con las particularidades de cada operación. 
- En el momento de la evaluación de las propuestas (mes 10), es necesario fijar los mecanismos de ajuste de precios, la capacidad de entrega y las alternativas financieras y, si la financiación se realizará por parte de una colocación pública de bonos, se requiere evaluación preliminar por parte de agencias de calificación crediticia.

- En el momento de la oferta final (BAFO -Best And Final Offer) (mes 14) se produce la aprobación definitiva de la operación por parte de del comité bancario, cuando la financiación sea mediante préstamo, y el compromiso de precios, en las operaciones financiadas con bonos.

- En el momento de la evaluación y nombramiento de los denominados Preferred Bidders (postores preferidos) (mes 22), por un lado, cuando haya sido requerida evaluación preliminar de agencias de rating, se les demandará la evaluación definitiva y, por otro, se realizará la evaluación de los mecanismos de ajuste de precios, capacidad de devolución y alternativas (bonos o préstamos alternativos) definitivas.

- En el momento de la firma del contrato (mes 25), se producirá el cierre de todas las operaciones contractuales y financieras: cierre financiero del proyecto, sindicación (en el caso -habitual- de participación colegiada de entidades financieras, garantías y acuerdos vinculados), cierre financiero y colocación de la emisión de bonos (razón por la que es en este momento cuando se manifiesta el riesgo de colocación de los mismos) y comenzará a tomar efecto cada uno de los compromisos establecidos durante el proceso.

\section{ANÁliSIS DE LOS RESULTADOS DE LA FASE PILOTO DE LA INI- CIATIVA DE BONOS PROYECTO}

Las metas fijadas para 2015 y 2020 constituyen etapas intermedias para los objetivos principales de la política europea de transportes, que se producirán en 2030 - a través de la denominada red central- y 2050 -a través de la red global. Ambas capas incorporan todos los modos de transporte con sus interconexiones y consisten en la infraestructura de transporte, aplicaciones telemáticas y medidas de acompañamiento (Pape, 2015, 2).

La primera evaluación de la iniciativa europea de bonos proyecto se realizó en base a los datos de junio de 2015 y fue publicada en abril de 2016. Para ello, se analizó la eficacia, eficiencia, relevancia y el valor añadido de la misma para aquellos que han estado involucrados en esta iniciativa (BEI, Comisión Europea y agentes directamente involucrados en proyectos vinculados a la iniciativa). Los principales datos de proyectos financiados en el marco de esta iniciativa se recogen en la Figura 8. 
Figura 8. Proyectos financiados en el marco de la iniciativa de bonos proyecto

\begin{tabular}{|c|c|c|c|c|}
\hline PROYECTO & PAÍS & FECHA & $\begin{array}{c}\text { IMPORTE } \\
\text { BONOS } \\
\text { PROYECTO } \\
\text { (millones) }\end{array}$ & ACTIVIDAD \\
\hline Castor & España & 2013 & $€ 1.400$ & $\begin{array}{c}\text { Almacenamiento } \\
\text { subterráneo de gas }\end{array}$ \\
\hline Greater Gabbard & Reino Unido & 2013 & GBP 305,1 & Energía eólica marina \\
\hline A11 & Bélgica & 2014 & $€ 577,9$ & $\begin{array}{c}\text { autopista PPP (acceso al } \\
\text { puerto de Zeebrugge) }\end{array}$ \\
\hline Axione & Francia & 2014 & $€ 189,1$ & $\begin{array}{c}\text { Conexión ultra } \\
\text { rápida banda ancha } \\
\text { (infraestructura digital) }\end{array}$ \\
\hline A7 & Alemania & 2014 & $€ 429,1$ & $\begin{array}{c}\text { autopista PPP } \\
\text { Bordesholm-Hamburgo }\end{array}$ \\
\hline $\begin{array}{c}\text { Gwynt y Mor } \\
\text { (GyM) }\end{array}$ & Reino Unido & 2015 & GBP 339,2 & $\begin{array}{c}\text { Energía eólica } \\
\text { Ronda 2A }\end{array}$ \\
\hline Puerto de Calais & Francia & 2015 & $€ 504$ & Puerto \\
\hline
\end{tabular}

Fuente: datos básicos EY (2015) y elaboración propia.

En este análisis es importante tener en cuenta varias cuestiones cuya influencia confluye para la obtención de los resultados que se han registrado y que se muestran en el informe final de evaluación (diciembre de 2015) de la Iniciativa Europa 2020 Project Bond (EY, 2015):

En términos de eficacia:

- Los bonos proyecto han impulsado a los inversores institucionales a participación en proyectos de infraestructuras.

- La iniciativa ha impulsado los mercados de deuda vinculados a proyectos nuevos en los sectores de transporte y energía en diferentes sectores y estructuras financieras.

En términos de eficiencia:

- La UE consiguió un efecto palanca de 22,83 de los recursos asignados a esta iniciativa (un coste total de los proyectos de 5.252 millones frente a los 230 de su aportación). Dado que inicialmente esperaba un multiplicador entre 15 y 20, la UE considera conseguidos sus objetivos.

- El análisis muestra que la participación de la UE ha contribuido al desarrollo de los mercados de capitales en relación con la financiación de proyectos de infraestructura de transporte y energía. 
En términos de relevancia:

- El diseño del instrumento, ha sido adecuado para las necesidades de los inversores en bonos, especialmente para proyectos nuevos.

- La relevancia del instrumento parece haber quedado condicionada al apetito de los inversores en el marco general, a situaciones puntuales de falta de liquidez y a los países considerados menos solventes.

En términos de valor añadido:

- Es necesario desarrollar nuevas medidas para alcanzar los objetivos del instrumento: incrementar la cartera de proyectos vinculados, racionalizar las iniciativas de la UE y centrarse en los proyectos centrales.

- Es necesario canalizar los esfuerzos hacia las áreas de mejora identificadas como estratégicas:

- Mejora de la regulación en materia de colaboración público privada y de procedimientos de autorización de iniciativas.

- Impulsar la capacidad de los Estados miembros para promover infraestructuras transnacionales.

Sin embargo, un análisis individualizado propio tanto del contexto como de las aportaciones y los proyectos financiados muestra que:

En términos generales:

- La situación del sistema financiero y su percepción en relación con los proyectos de infraestructuras han cambiado sustancialmente durante los últimos años. Se ha recuperado la liquidez del sistema y los tipos de interés han vuelto a niveles que hacen sostenibles proyectos que no lo eran hace 6 años, en parte por la reducción de la prima de riesgo aplicable a los mismos.

- Dado que el modelo de esta iniciativa constituye un paquete de dos medidas complementarias (aportación del BEI y colocación de bonos proyecto), no resulta fácil conocer cuál de las dos ha constituido un mayor incentivo para la participación de los inversores privados.

En términos de eficacia:

- Solo 2 de los 7 proyectos financiados fueron nuevos, es decir, con riesgo de construcción, por lo que consideramos que no se ha conseguido ese objetivo de dinamizar los mercados de valores en relación con los proyectos nuevos, al menos en un grado suficiente. 
- Es importante, además, destacar el hecho de que en el objetivo de conseguir la financiabilidad de proyectos por vías alternativas, debe estudiarse su calificación crediticia. Así, de los 7 proyectos, únicamente 3 no serían financiables per se: los de nueva creación y el proyecto Castor ${ }^{21}$, que incluso siendo una instalación preexistente, contaba con una calificación crediticia inferior a la considerada habitualmente como financiable por las entidades financieras tradicionales (A-).

\section{Figura 9. Características y financiabilidad de los proyectos que han contado con bonos proyecto}

\begin{tabular}{|c|c|c|}
\hline Proyecto & $\begin{array}{c}\text { Tipo } \\
\text { (Nueva construcción o } \\
\text { Preexistente) }\end{array}$ & $\begin{array}{c}\text { Financiabilidad del proyecto } \\
\text { per se? }\end{array}$ \\
\hline Castor & Preexistente & No \\
\hline Greater Gabbard & Preexistente & No \\
\hline A11 & Nuevo & Sí \\
\hline Axione & Preexistente & No \\
\hline A7 & Nuevo & Sí \\
\hline GyM & Preexistente & Sí \\
\hline Puerto de Calais & Preexistente & \\
\hline
\end{tabular}

Fuente: datos básicos EY (2015) y elaboración propia.

En términos de eficiencia:

- Una de las modalidades (financiada) no ha tenido ningún seguimiento. Todos los proyectos elegibles han recurrido a las garantías que supone la modalidad no financiada.

- El efecto multiplicador se ha conseguido en los proyectos de la red de energía (270 frente a la media de 22,83), donde la aportación de la UE ha sido más reducida (ver Figura 10).

- Cinco de los siete proyectos financiados han sido preexistentes (brownfield), lo que muestra la reducida utilidad como instrumento, con el enfoque actual, para impulsar la creación de nuevas infraestructuras.

- Aunque la colaboración institucional es importante, la aportación de los fondos de la UE no ha sido determinante en ninguno de los proyectos, dadas las cifras que se manejaron para su financiación.

\footnotetext{
${ }^{21}$ Además, debe tenerse en cuenta que el proyecto Castor fue paralizado en 2014, ya que su puesta en marcha provocó terremotos de pequeña magnitud en el entorno.
} 
Figura 10. Proyectos en el marco de la iniciativa de bonos proyecto y financiación recibida

\begin{tabular}{|l|c|c|c|c|}
\hline \multicolumn{1}{|c|}{$($ millones $€$ ) } & UE & BEI (PBCE) & BONOS & $\begin{array}{c}\text { COSTE TOTAL } \\
\text { DEL PROYECTO }\end{array}$ \\
\hline $\begin{array}{l}\text { Red Transeuropea } \\
\text { de Transporte }\end{array}$ & 200 & 250 & 1.511 & 2.293 \\
\hline $\begin{array}{l}\text { Red Transeuropea } \\
\text { de Energia }\end{array}$ & 10 & 324 & 2.223 & 2.702 \\
\hline $\begin{array}{l}\text { Tecnologías } \\
\text { de la Información } \\
\text { y las Comunicaciones }\end{array}$ & 20 & 38 & 189 & 257 \\
\hline TOTAL & 230 & 612 & 3.700 & 5.252 \\
\hline
\end{tabular}

Fuente: datos básicos EY (2015) y elaboración propia.

En términos de relevancia:

- Se ha comprobado cómo el proceso diseñado para determinar la elegibilidad de los proyectos no ha funcionado correctamente. Únicamente se registraron 7 solicitudes y todas resultaron positivas, en un contexto de fuertes necesidades de infraestructuras, por lo que las dificultades administrativas para culminar la fase inicial de planteamiento y propuesta han sido un obstáculo para el cumplimiento de su objetivo operativo.

- Más aún, únicamente un proyecto (Castor) ha sido capaz de atraer recursos que no habrían sido conseguidos por las vías alternativas tradicionales, por lo que no consideramos que se hayan cubierto las necesidades de los inversores.

- Un instrumento transnacional cuya utilidad se limita a situaciones puntuales de falta de liquidez o para países con problemas de solvencia no cumple los objetivos generales con que fueron diseñados los bonos proyecto.

En términos de valor añadido:

- Es clave trabajar para reducir la carga administrativa necesaria para estas iniciativas transnacionales y mejorar su transparencia. No es posible que se produzcan cuellos de botella en el proceso de elegibilidad de los proyectos, para lo que es necesaria una buena comunicación y transparencia.

- Los proyectos prioritarios para la UE lo son en términos de retorno social y no siempre cuentan con una rentabilidad financiera atractiva. En este caso, proyectos necesarios para la sociedad pero que generan ingresos insuficientes y que, sin embargo, requieren de inversiones millonarias, pueden ser descartados por no alcanzar la calificación crediticita exigida. Además, 
desde un punto de vista teórico, todos los países de la UE tienen derecho a poder ser elegibles para financiar sus proyectos mediante el mecanismo de los bonos proyecto. Sin embargo, desde un punto de vista práctico, no parece lógico que se pueda desligar el riesgo soberano del proyecto, lo que genera diferencias sustanciales entre países de la UE a la hora de poder ser receptores de esta alternativa de financiación.

En cualquier caso, una vez superada la prueba piloto, el mercado se encuentra preparado para valorar este nuevo instrumento de inversión (Moody's, 2016: 4) sin los recelos previos, propios de la incertidumbre que acarrea la gestión de riesgos en una situación sin compromiso firme de las instituciones.

\section{CONCLUSIONES}

1. Las infraestructuras son un instrumento generador de riqueza y un importante estabilizador regional y social. Por este motivo, la Comisión Europea y el BEI están contribuyendo a apoyar este tipo de proyectos mediante la absorción de algunos de los riesgos que de lo contrario recaerían en acreedores preferentes, lo que desincentivaría la inversión privada. El papel del BEI consiste en ayudar a desarrollar y ampliar una cartera de proyectos PPP elegibles por sus características intrínsecas y por su alineamiento con el mapa estratégico europeo de transporte. En cualquier caso, la colaboración es clave: por un lado, entre instituciones; y por otro, entre instituciones públicas y agentes privados, para la consecución de objetivos que favorecen a todos ellos.

2. La UE creó un conjunto de iniciativas "innovadoras" para impulsar la financiación de proyectos de infraestructuras: el fondo Marguerite, el Instrumento de Garantía de Crédito para Proyectos de la Red Transeuropea de Transporte y los bonos proyecto. Estos últimos han sido la apuesta más fuerte -por considerar que cuentan con un mayor potencial para captar recursos, por su naturaleza y por el compromiso presupuestario asociado- para facilitar la colaboración público-privada, proporcionando garantías que mejorarán la calidad crediticia de la deuda senior para proyectos de infraestructuras de alto interés estratégico europeo. Los bonos proyecto proporcionan garantías que mejoran la calidad crediticia de la deuda, atrayendo a capitales privados, principalmente fondos de pensiones y compañías de seguros, lo que agiliza la puesta en marcha de los proyectos de infraestructura y reduce los costes de financiación.

3. Aunque desde el momento de la creación de estos instrumentos, la liquidez en el sistema era muy reducida y este punto ha cambiado en los últimos años, siguen observándose reticencias por parte de los bancos a financiar proyectos nuevos. 
4. Sin embargo, los bonos proyecto que la UE considera innovadores no lo son en realidad, ya que existen instrumentos similares en otras partes del mundo. Por ejemplo, están presentes en Estados Unidos desde la Transportation Infrastructure Finance and Innovation Act (TIFIA) en 1998, que proporcionan préstamos directos, garantías a préstamos y líneas de crédito para proyectos nacionales o regionales de transporte terrestre. O los Private Activity Bonds (PABs), bonos exentos de impuestos, emitidos por o en nombre de la administración (local o estatal) con el propósito de proporcionar beneficios especiales para la financiación de proyectos de interés. Este tipo de bonos se emplean también en Canadá y Sudamérica.

5. La financiación mediante bonos proyecto pretende alcanzar la viabilidad financiera de los proyectos: 1) haciendo fluir el crédito hacia los proyectos de infraestructuras; 2) alargando los plazos de financiación para los proyectos; 3) reduciendo el precio de los recursos financieros. Sin embargo, pueden resultar un mecanismo insuficiente, especialmente en los greenfield, dado que pocos proyectos pueden acceder a este mecanismo por los exigentes requisitos exigidos por el BEI. Los bonos proyecto han financiado hasta 2015 un total de 7 proyectos, de los cuales tan solo dos fueron nuevos.

6. Sin embargo, los bonos proyecto en su definición actual han demostrado impulsar la financiabilidad únicamente en contadas ocasiones (como ocurrió en España con el proyecto Castor). En términos generales no han servido para hacer financiables a proyectos que no lo eran mediante otros instrumentos, debido a un perfil de riesgo elevado. Por tanto, hasta ahora, su éxito para dinamizar la inversión europea en infraestructuras estará asociado a su utilización combinada con otro tipo de financiación, incluida la bancaria, con garantías, tanto públicas como privadas y con incentivos fiscales a los proyectos.

7. El hecho de que hasta el momento todos los proyectos presentados fuesen elegibles (y su reducido número) demuestra el necesario replanteamiento de los mecanismos y los criterios de elegibilidad. De hecho, ya se están dando los primeros pasos (Comisión Europea, 2015) en la redefinición de los antes denominados proyectos prioritarios (y ahora red básica-core network).

8. Europa se enfrenta a una enorme necesidad de inversión en infraestructuras, y los bonos proyecto, con la definición actual, aún no constituyen un instrumento eficaz para atraer la suficiente financiación procedente de los mercados de capitales. Por este motivo, será fundamental re-diseñar el modelo con la colaboración de los potenciales interesados: bancos privados, inversores institucionales y empresas de seguros para conseguir un diseño de interés para todos los agentes interesados.

9. Los proyectos de infraestructuras tienen un impacto estructural y social tan importante que no pueden dejarse únicamente a los designios de los 
mercados de capitales y su análisis de riesgo y rentabilidad. Por este motivo, las instituciones públicas deben tomar un papel activo e impulsar con fuerza un marco legal favorable y unos criterios claros para la generación de una infraestructura transnacional amplia y eficaz, en beneficio de todos los ciudadanos de la Unión.

10. La colaboración entre instituciones es fundamental para el éxito de estos proyectos internacionales, pero será necesaria la especialización de cada una de ellas para conseguir resultados óptimos. Así, sería positivo enfocar la aportación financiera de la UE a las etapas iniciales de los proyectos, de forma que sí sea decisiva en la fase de propuesta e iniciación debido a: 1) la canalización de propuestas en la línea de los objetivos de las distintas redes transnacionales; 2) la financiación de los estudios preliminares que conducen a decidir acerca de la elegibilidad de los proyectos; 3 ) el enfoque de la institución hacia una fase clave cuyo desarrollo ha demostrado ser ineficiente a lo largo del piloto.

11. Así, en esta línea de colaboración institucional, será necesario hacer el seguimiento del Fondo Europeo para Inversiones Estratégicas (FEIE), creado en 2015, cuya finalidad es impulsar el crecimiento económico y la competitividad a largo plazo, mediante la movilización de recursos públicos (a cargo del presupuesto de la UE) para atraer la inversión privada y con el triple objetivo de: 1) mejorar la capacidad de absorción de riesgos de las operaciones de inversión del BEI; 2) hacer frente a las disfunciones del mercado y a las situaciones de reducida inversión, 3) impulsar la inversión (315 000 millones de euros adicionales en tres años).

\section{BIBLIOGRAFÍA}

BANCO EUROPEO DE INVERSIONES (2016), Investment Plan for Europe. Banco Europeo de Inversiones. Luxemburgo. http://www.eib.org/projects/ cycle/ (última consulta 08/06/2016).

BANCO EUROPEO DE INVERSIONES (2012), An outline guide to Project Bonds Credit Enhancement and the Project Bond Initiative. Banco Europeo de Inversiones. Luxemburgo.

BANCO MUNDIAL (1994), "Valuing Infrastructure Stocks and Gains from Improved Performance", Background paper prepared for World Bank, World Development Report.

CHAMPION, J. (2016), Research Briefing. Trans-European Transport Network (TEN-T) - a quick guide. National Assembly for Wales Commission. Research Service National Assembly for Wales. 
COMISIÓN EUROPEA (2015a), Making the best use of new financial schemes for European transport infrastructure projects. Bruselas. http://ec.europa.eu/ transport/themes/infrastructure/ten-t-guidelines/doc/2015_06_03_cbs_ action_plan_final.pdf (última consulta: 15/06/2016).

COMISIÓN EUROPEA (2015b), Informe de la Comisión al Parlamento Europeo y al Consejo de evaluación de las finanzas de la Unión basado en los resultados obtenidos. Bruselas, 26.6.2015 COM(2015) 313 final.

COMISIÓN EUROPEA (2011), Libro Blanco. Hoja de ruta hacia un espacio único europeo de transporte: por una política de transportes competitiva y sostenible. Luxemburgo. http://ec.europa.eu/transport/themes/strategies/ doc/2011_white_paper/white-paper-illustrated-brochure_es.pdf (última consulta: 15/06/2016).

COMISIÓN EUROPEA (2012), Connect to compete, http://ec.europa.eu/ transport/facts-fundings/connect-to-compete/index_en.htm (última consulta 08/06/2016).

COMISIÓN EUROPEA. (2012), Transport modes, http://ec.europa.eu/transport/modes/rail/news/shift-to-rail_en.htm (última consulta 06/07/2016).

DUPAS, N. y otros (2011). Mini-perm Structures in PPP Contracts: Risks and Opportunities. https://halshs.archives-ouvertes.fr/halshs-00686701/ document (última consulta: 10/06/2016).

EPEC y FRESHFIELDS BRUCKHAUS DERINGER (2013), Financing PPPs with project bonds in Germany. An analysis of procurement issues. http:// www.eib.org/epec/resources/financing-ppps-project-bonds-in-germanyjuly-2013pdf1.pdf (última consulta 01/06/2016)

EY (2015), Ad-hoc Audit of the pilot phase of the Europe 2020 Project Bond Initiative. Comisión Europea.

FRESHFIELDS BRUCKHAUS DERINGER LLP. (2012), From policy to proof of concept, and beyond: Outlook for infraestructure 2012.

GEREBIZZA, E. y TRICARICO, A. (2013), Large infrastructure to overcome the crisis? The hidden risks of the Europe 2020 project bond initiative.

GROUT, P. A. (2003), "Public and private sector discount rates in PublicPrivate Partnerships", The Economic Journal, num. 113, pp. 62-68.

MARGUERITE (2016), The PCI initiative: how the Marguerite Fund can help this succeed.

MOODY'S (2016), Moody's: Europe's project bond market set for growth after successful pilot. Moody's Investors Service.

OCDE (2007), Infrastructure to 2030, vol. 2. Mapping policy for electricity, water and transport.

PAPE, M. (2015), An overview of the Trans-European Transport Network. Servicio de Estudios del Parlamento Europeo. 
Eva Ballesté Morillas, Rodrigo Martín García y Ma Teresa Corzo Santamaría

PWC (2014), European Commission DG Transport and Mobility. Ad-hoc audit of the Marguerite Fund. Executive Summary. Comisión Europea

ROSSI, E. y otros (2015), Infrastructure project finance and project bonds in Europe. Springer.

SPACKMAN, M. (2002), "Public-private partnerships: lessons from the British approach”, Economic Systems, Vol. 26, num. 4, pp. 283-301. 